\title{
COMMENT
}

\section{Comment on 'Non-equilibrium thermodynamics of light absorption'}

\author{
David L Andrews \\ School of Chemical Sciences, University of East Anglia, Norwich NR4 7TJ, UK
}

Received 7 May 1999, in final form 1 November 1999

\begin{abstract}
A recent paper by Meszéna and Westerhoff (1999 J. Phys. A: Math. Gen. 32 301) has aimed to address what is referred to as a principal question of biological thermodynamics, the possibility of describing photosynthesis in terms of non-equilibrium thermodynamics. The issue is associated with a misrepresentation of the fundamental photophysics involved, and as a result the analysis is invalid.
\end{abstract}

Over two decades have elapsed since Hill [1] elegantly summarized the many constraints involved in describing photosynthesis in terms of conventional non-equilibrium thermodynamics. During the intervening period, the fundamental science of the process has made enormous advances, some earlier concepts being modified and others abandoned [2]. The current understanding of the basic principles has now been in place for some considerable time, and the recent analysis by Meszéna and Westerhoff [3] (MW) incorrectly reflects the photophysics involved.

Although in the abstract MW misrepresent 'the very first step' in photosynthesis as the conversion of solar energy to the free energy of living material, the paper correctly employs the descriptor for the initial photoabsorption by chlorophyll. However, the analysis fails to account for the fact that the molecules which absorb sunlight are not responsible for photosynthetic energy conversion - the latter process takes place at a physically separate reaction centre. For this reason alone, the conclusion that 'light can now be viewed as a substrate of the photosynthetic reaction centre' is not realizable. In photosynthesis, photon energy absorbed by antenna molecules transfers with extreme rapidity to the reaction centre [4] and in the reported equilibrium,

$$
\gamma+\mathrm{Chl} \leftrightarrow \mathrm{Chl}^{*}
$$

the disappearance of the product species and associated energy dissipation by resonance energy transfer is far more significant than through the reverse reaction. In green plants, two types of photosystems (PS) initiated by the forward reaction of (1) together generate the redox potential for oxidation of water. Each involves pigment species $P$ with different spectral characteristics: photoabsorption is centred at around $700 \mathrm{~nm}$ in PS I, and around $680 \mathrm{~nm}$ in PS II. In PS I, the primary processes following (1) can be summarized as [5]:

$$
\begin{aligned}
& \mathrm{Chl}^{*}+\mathrm{Chl} \rightarrow \mathrm{Chl}+\mathrm{Chl}^{*} \\
& \mathrm{Chl}^{*}+P 700 \rightarrow \mathrm{Chl}+P 700^{*} \\
& \left(P 700^{*} A\right) \rightarrow\left(P 700^{+} A^{-}\right) .
\end{aligned}
$$


Equation (2) represents Förster excitation transfer over a series of chlorophyll molecules, energy eventually transferring to the reaction centre, equation (3). The ensuing electron transfer (4) to an acceptor $A$ produces a reducing agent $A^{-}$; a similar scheme in PS II, based on $P 680$ chlorophyll, results in the formation of an oxidant $P 680^{+}$. Subsequent redox reactions lead to the four-electron oxidation of water and four-electron reduction of carbon dioxide in the reaction centre. The losses (manifest as heat) associated with the redox sequence favour the required directionality of the process [4].

On detailed photonic issues, MW's statement that 'no time reversal of induced emission exists' is wrong and curiously asserted, since their equations (9) and (13) correctly reflect microscopic time reversal. As spin-1 fundamental particles, photons are subject to Bose statistics, and for a photon mode with annihilation operator $a$ and creation operator $a^{+}$, the result

$$
\left\langle n+1\left|a^{+}\right| n\right\rangle=\langle n|a| n+1\rangle^{*}
$$

is directly related to the fundamental boson commutation relation $\left[a, a^{+}\right]=1$ and, moreover, associated with the zero-point energy of the vacuum field. In this sense some of the statements following MW's equation (13) lead to self-contradiction. It is precisely because quantum electrodynamics produces a result where the probability of photon emission is proportional to the number of photons after the transition that it succeeds, where semiclassical theory fails, in explaining spontaneous emission, etc [6]. Where photon absorption and emission are concerned, apparent irreversibility emerges as an issue only in connection with the density of radiation states for the latter.

Several major problems with the development of theory by MW concern giving insufficient heed to either the intensity or the detailed spectral character of sunlight. The mean irradiance $I$ of sunlight on the Earth's surface is $1.4 \mathrm{~kW} \mathrm{~m}^{-2}$; its spectral composition is largely congruent with a black body temperature of $5762 \mathrm{~K}$. The detailed profile is modified through attenuation by atmospheric gases [7]. Because of the energy dissipation from the absorber chlorophyll (2), the radiation-absorber system is not a closed one, and thermodynamic arguments that relate to black body radiation are inapplicable. A chlorophyll molecule absorbing sunlight is not in thermodynamic equilibrium with the Sun. The case $T=T_{r}$, considered by MW at several junctures, corresponds to the chemically and biologically unreasonable situation of a leaf at the temperature of the Sun itself.

Again, there is no meaningful prospect of more than one photon in a given radiation mode interacting with one molecule of chlorophyll. Suppose we disregard the spectral span of sunlight, and consider all its energy to be incident at some mean frequency $\bar{v}=3.5 \times 10^{14} \mathrm{~Hz}$ [8]. The instantaneous probability of a chlorophyll (whose porphyrin chromophore occupies a volume $V \sim 10^{-27} \mathrm{~m}^{3}$ ) being intercepted by a single photon can be estimated from the Planck relation, from which the mean number of photons, $\langle n\rangle \sim I V / h c \bar{v}$, is about $2 \times 10^{-14}$. The number of photons in any specific mode is much smaller, and there is no meaningful possibility of populating the $n=2,3$ or 4 levels shown in MW's figure 1. Furthermore, the probability of a second photon arriving before the excitation resonantly transfers away from an initially excited chlorophyll is governed by the mean interval between photons, $\tau=h v / I A$. For a chromophore presenting a maximum cross section $A \sim 2 \times 10^{-18} \mathrm{~m}^{2}$ to orthogonal radiation, we find $\tau \sim 80 \mu \mathrm{s}$, considerably in excess of the picosecond timescale for energy transfer. The fact that in photosynthetic applications we always have $\langle n\rangle \ll 1$ means there is no physical basis for considering the behaviour as $\langle n\rangle \rightarrow \infty$. Postulate 1, concerning the activity of the 'photon gas', is redundant, and so too is postulate 2 concerning the 'infinite series of emission/absorption processes' wherein one or more photons are added to both sides of process (1). 
In conclusion there is nothing singular about the application of quantum electrodynamics to the photon absorption process involved in photosynthesis. It is not necessary or advisable to employ unfamiliar concepts such as regarding 'Fock states ... as the chemical agents'. If there is any meaning to such a proposal, the paper by MW has not advanced its acceptance.

\section{Acknowledgments}

I am grateful to Brian Robinson, Moises Silbert and Geoff Stedman for comments on this manuscript.

\section{References}

[1] Hill T L 1977 Free Energy Transduction in Biology (New York: Academic) pp 213-21

[2] Hall D O and Rao K K 1994 Photosynthesis 5th edn (Cambridge: Cambridge University Press) p xiii

[3] Meszéna G and Westerhoff H V 1999 J. Phys. A: Math. Gen. 32301

[4] van Grondelle R and Somsen O J G 1999 Excitation energy transfer in photosynthesis Resonance Energy Transfer ed D L Andrews and A A Demidov (New York: Wiley) pp 366-98

[5] Andrews D L 1997 Lasers in Chemistry 3rd edn (Berlin: Springer) pp 188-90

[6] Craig D P and Thirunamachandran T 1998 Molecular Quantum Electrodynamics (New York: Dover) pp 16, 94

[7] http://dosxx.colorado.edu/ kachun/graphics/solar_spect_at_earth.gif

[8] Lynch D K and Soffer B H 1999 Opt. Photonics News March 28-30 